\title{
Interrogation System Based on "Figure-of-Eight" Fiber Loop Mirror
}

\author{
Ricardo M. Silva ${ }^{\mathrm{a}, \mathrm{b} *}$, A. Layeghi ${ }^{\mathrm{c}}$, M. I. Zibaiic ${ }^{\mathrm{c}}$ H. Latific ${ }^{\mathrm{c}}$ J. L. Santos ${ }^{\mathrm{a}, \mathrm{b}}$, \\ A. B. Lobo Ribeiro ${ }^{\text {, }}$, and O. Frazão ${ }^{a}$ \\ ${ }^{a}$ INESC Porto, Rua do Campo alegre 687, 4169-007 Porto, Portugal \\ ${ }^{\mathrm{b}}$ Fac. de Ciências da Universidade do Porto, Rua do Campo Alegre 687, 4169-007 Porto, Portugal \\ ${ }^{\mathrm{c}}$ Laser \& Plasma Research Institute, Shahid Beheshti University, Evin, Tehran, Iran. \\ ${ }^{\mathrm{d}}$ Faculty of Heath Sciences, University Fernando Pessoa, Rua Carlos da Maia 296, 4200-150 Porto, Portugal
}

\begin{abstract}
A theoretical and experimental study of a new fiber loop mirror based on a "figure-of-eight"configuration, is reported. For the theoretical model, the Jones matrix analysis is analyzed. The configuration is tested as an interrogation system where the spectral response arises from the combination of the reference signal modulated by the sensor signal. The configuration is characterized in mechanical strain and presents a phase sensitivity of $8.2 \mathrm{mrad} / \mu \varepsilon$.
\end{abstract}

Keywords: fiber optic sensors, Fiber loop mirror.

\section{INTRODUCTION}

Fiber Loop Mirrors (FLMs) are attractive devices to be used in sensing applications [1]-[3]. An FLM, two optical waves propagate in opposite directions using the two output ports of a $2 \times 2$ fiber coupler connected together. All light is reflected back to the input port. FLMs can have inside of the loop an highly birefringent fiber (HiBi-FLM which presents several advantages, such as, input polarization independence and high extinction ratio. Besides the gyroscope application, various kinds of fiber sensors based on FLMs have been fabricated [3], such as temperature sensors [2], [4], strain sensors [5], [6], pressure sensors, liquid level sensors [7], biochemical sensors, UV detection [8] and multiparameter measurement [9]-[10]. Recently, three different high-birefringent fiber loop mirrors were analyzed theoretically and experimentally [11]. All these three configurations can be applied in specify sensing applications, however one of them can be used simultaneously as a sensor and as an interrogation system.

This work presents a theoretical and experimental study of a new fiber loop mirror based on a "figure-of-eight" configuration. For the theoretical model, the Jones matrix formalism is used. The configuration is tested as an interrogation system where the spectral response arises from the combination of the reference signal modulated by the sensor signal.

\section{THEORETICAL MODEL}

In order to investigate the novel FLM interferometer based on a "figure-of-eight" implemented by a $3 \times 3$ ports fiber coupler, a theoretical characterization of the transmission property of the fiber loop interferometer is necessary and this is based on the schematic diagram shown in figure 1.

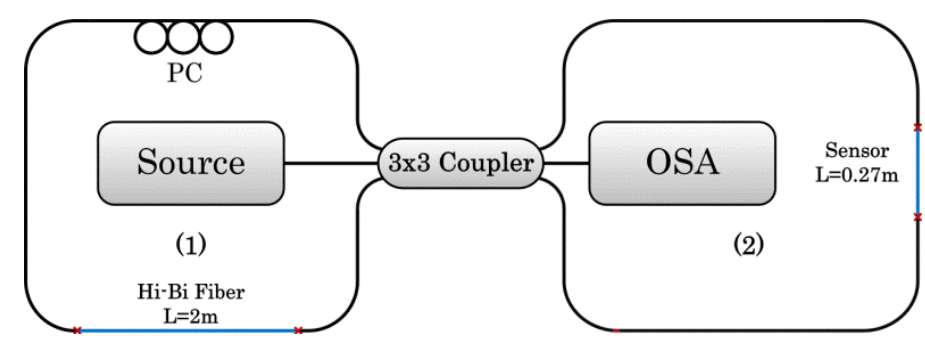

Figure 1. Experimental setup.

\footnotetext{
*rmsilva@inescporto.pt; phone: (+351) 220402 301; fax: (+351) 220402437
} 
The proposed setup consists of a broadband $3 \times 3$ fiber coupler and a length of Hi-Bi fiber spliced in both loops. This interferometer has different interference periods due to Hi-Bi fiber lengths. To simplify the analysis it is assumed that the excess loss of the coupler is negligible for the fundamental mode propagation. We also assume that the coupling effect of the fiber coupler is sufficiently localized such that the physical length of the coupling section of the coupler may be neglected. These simplifications allow us to restrict the consideration of the longitudinal field variations to points immediately before and after the coupling region. As seen in Figure 1, the broadband optical source at the input port of the $3 \times 3$ coupler illuminates the system, and its light is divided into three optical beams by $100 / 3$ power ratio. According to the setup, two of the three light beams propagate in the clockwise and counter clockwise directions, respectively, and meet again in the coupler to split into three beams. Two of the three beams counter-propagate on the second loop. Light beams meet again in the coupler to induce interference. Due to the fiber loss the light circulation in the fiber for the second time is ignored. The interfered beams after the $3 \times 3$ coupler also split to propagate into the output port and input port, respectively. The characteristics of these interference beams are determined by the birefringence of the Hi-Bi fiber itself and by the splicing angle rotation of Hi-Bi fibers (or the polarization state of the $P$ ) simultaneously. Using the schematic explanation of the loop based on the $\mathrm{Hi}-\mathrm{Bi}$, we consider the propagation direction of the light in the loop as shown in figure 2.

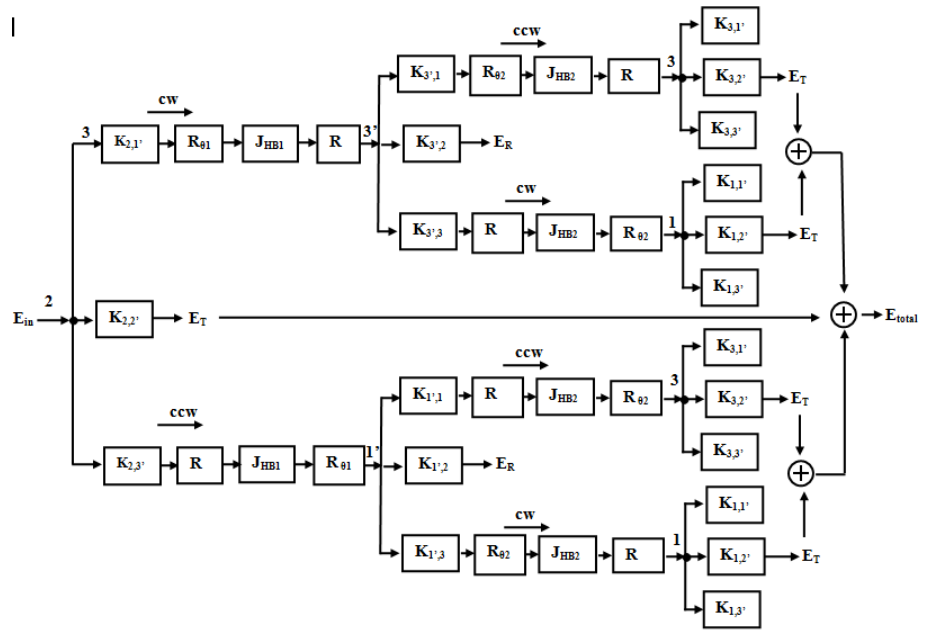

Figure 2. Equivalent optical circuit of the proposed two loops interferometer based on the Hi-Bi.

The characteristics of these interference light beams are determined by the birefringence value of the Hi-Bi and splicerotation of Hi-Bi fiber (the polarization state of the PC) simultaneously. Using a similar method to our previous analysis of the transmission field [11], we can derive Jones matrix analysis of the transmission field, $E_{\text {transmission }}$, expressed as:

$$
\begin{aligned}
& E_{\text {transmission }}=\left(\left\{\left(\left[K_{12^{\prime}}\right]\left[R_{\theta 2}\right]\left[J_{H B 2}\right][R]\left[K_{3^{\prime} 3}\right]\right)+\left(\left[K_{32^{\prime}}\right][R]\left[J_{H B 2}\right]\left[R_{\theta 2}\right]\left[K_{3^{\prime} 1}\right]\right)\right\} \times\right. \\
& \left([R]\left[J_{H B 1}\right]\left[R_{\theta 1}\right]\left[K_{21^{\prime}}\right]\right)+\left\{\left(\left[K_{12^{\prime}}\right]\left[R_{\theta 2}\right]\left[J_{H B 2}\right][R]\left[K_{1^{\prime} 3}\right]\right)+\left(\left[K_{32^{\prime}}\right][R]\left[J_{H B 2}\right]\left[R_{\theta 2}\right]\left[K_{1^{\prime} 1}\right]\right)\right\} \times \\
& \left.\left(\left[R_{\theta 1}\right]\left[J_{H B 1}\right][R]\left[K_{23^{\prime}}\right]\right)+\left[K_{22^{\prime}}\right]\right) E_{\text {in }}
\end{aligned}
$$

where $\left[K_{i j}\right]$ is the coupling matrix of the $3 \times 3$ fiber coupler. In an ideal $3 \times 3$ coupler, there is a $120^{\circ}$ phase difference between any two of the three ports. The average phase differences between two of the three outputs measured experimentally and it shows that the assumption of this difference phase is coincident with experiment. These matrices are given as

$$
\begin{aligned}
& {\left[K_{11^{\prime}}\right]=\left[K_{12^{\prime}}\right]=\left[K_{13^{\prime}}\right]=\left[K_{21^{\prime}}\right]=\left[K_{31^{\prime}}\right]=\frac{1}{\sqrt{\alpha}}\left[\begin{array}{ll}
1 & 0 \\
0 & 1
\end{array}\right]} \\
& {\left[K_{23^{\prime}}\right]=\left[K_{32^{\prime}}\right]=\frac{\exp (-i 2 \pi / 3)}{\sqrt{\beta}}\left[\begin{array}{ll}
1 & 0 \\
0 & 1
\end{array}\right]}
\end{aligned}
$$




$$
\left[K_{22^{\prime}}\right]=\left[K_{33^{\prime}}\right]=\frac{\exp (i 2 \pi / 3)}{\sqrt{\gamma}}\left[\begin{array}{ll}
1 & 0 \\
0 & 1
\end{array}\right]
$$

It is clear $K_{i j}=K_{j i}$. That $\alpha, \beta$ and $\gamma$ are the splitting ratio of coupler for asymmetric coupler, here it is assumed $\beta=\gamma=2.94$ and $\alpha=5.125$, approximately according to experimental result of $3 \times 3$ coupler. While in symmetric coupler, $\alpha, \beta$ and $\gamma$ are equal. The matrix $[R]$ is a coordinate conversion matrix due to the folded fiber in the loop. It is given as

$$
[R]=\left[\begin{array}{cc}
-1 & 0 \\
0 & 1
\end{array}\right]
$$

Next, $\left[J_{H B}\right]$ is the Jones matrix of the Hi-Bi fiber, which is expressed as

$$
\left[J_{H B}\right]=\left[\begin{array}{cc}
e^{j \beta_{e} L} & 0 \\
0 & e^{j \beta_{o} L}
\end{array}\right]
$$

Here, the propagation constants along the $e$-axis and $o$-axis of the Hi-Bi fibers are represented by $\beta_{e}$ and $\beta_{o}$, respectively, and the length of the $\mathrm{Hi}-\mathrm{Bi}$ fiber is expressed as $L$. Based on the birefringence of the Hi-Bi fiber, that is, $n_{e o}=n_{e}-n_{o}$, the difference of propagation constants, $\beta_{e}-\beta_{o}$, is equal to $2 \pi n_{e o} / \lambda$. The last $\left[R_{\theta}\right]$ is the Jones matrix of the rotation. The Jones matrix is expressed as

$$
R_{\theta}=\left[\begin{array}{cc}
\cos (\theta) & \sin (\theta) \\
-\sin (\theta) & \cos (\theta)
\end{array}\right]
$$

Where $\theta$ is the orientation of the axes of the wave plate with respect to the laboratory coordinates.

\section{EXPERIMENTAL RESULTS}

Figure 3 shows the theoretical simulation and the experimental the fringe patterns of the sensor signal combined with the reference signal. Two different frequencies are observed. The higher frequency corresponds to the reference signal and has a wavelength periodicity of $3 \mathrm{~nm}$. The lower frequency, shown from the amplitude modulation of the reference signal, was due to the sensor response. For mechanical strain characterization, the sensing head was attached to a translation stage with a step movement resolution of $1 \mu \mathrm{m}$. Using the variation of two peaks, it was possible to reconstruct the fringe of the sensor signal. In this case, the two peaks in quadrature were enough to measure the phase variation of the sensor. The normalized amplitude outputs represented in Figure 4 were obtained from both peaks. Besides the amplitude variation of the peaks when strain was applied, their curve fittings are also represented.
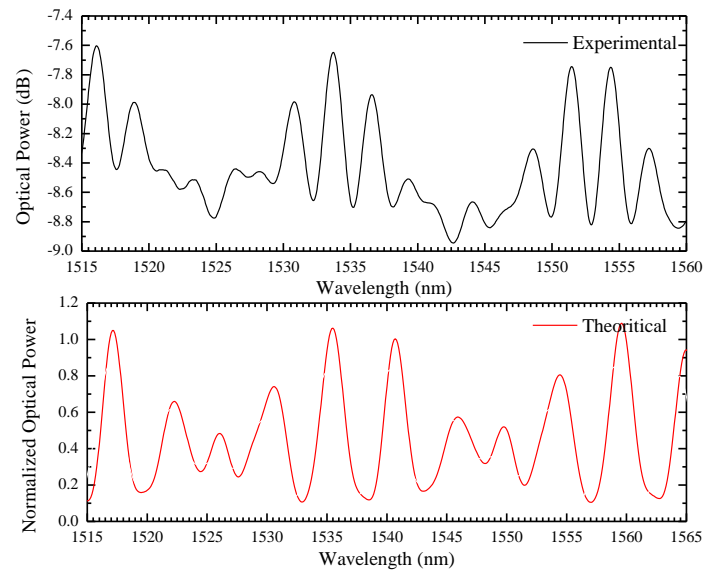

Figure 3. Theoretical and experimental response of the sensing head.

A quadrature phase-shifted relation can be observed between these two signals, which allows the phase recovery from $\phi=\tan ^{-1}\left(P_{1 n} / P_{2 n}\right)$. Where $P_{1 n}$ and $P_{2 n}$ are the normalized optical powers of $P_{1}$ and $P_{2}$, respectively [12]. The strain induced phase change is shown in Fig. 5. Mechanical strain was applied between 0 and $600 \mu \varepsilon$, resulting in a linear slope with a sensitivity of $8.2 \mathrm{mrad} / \mu \varepsilon$. 


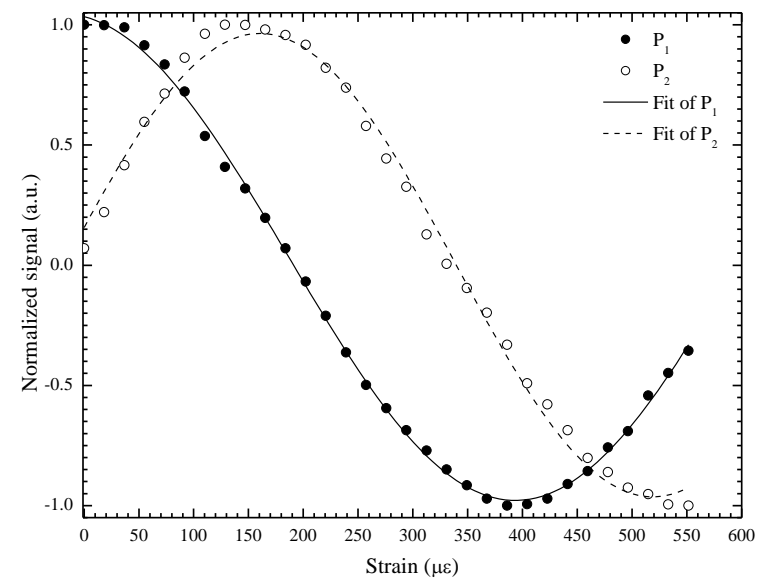

Figure 4. Normalized output signals versus applied mechanical strain, and the correspondent curve fitting of the experimental data.

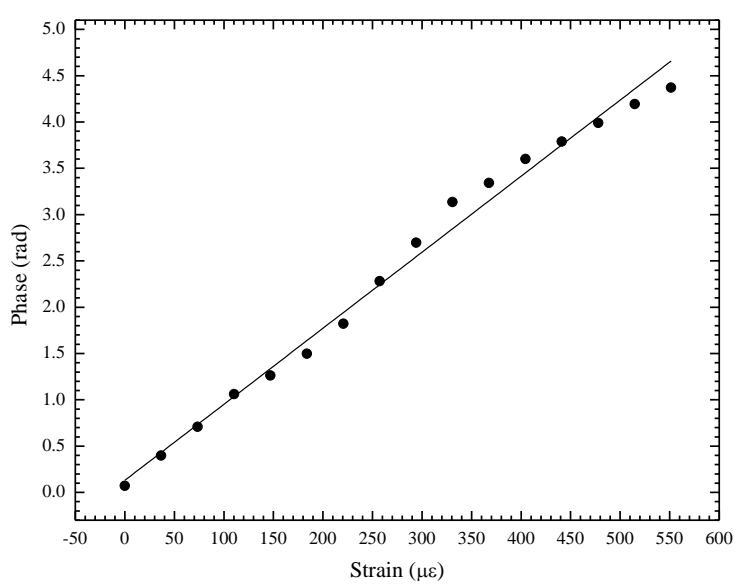

Figure 5. Strain measurement using the phase recovery.

\section{CONCLUSIONS}

Summarizing, a theoretical and experimental studied for a new fiber loop mirror based on a "figure-of-eight" made using a $3 \times 3$ port fiber coupler is demonstrated. The theoretical model based on Jones matrix formalism was studied and presents similar behavior when compared with the experimental fringe pattern. The configuration was tested as an interrogation system where the spectral response arises from the combination of the reference signal modulated by the sensor signal. The configuration was characterized in strain and presents a phase sensitivity of $8.2 \mathrm{mrad} / \mu \varepsilon$.

\section{REFERENCES}

[1] Mortimore, D. B., "Fiber Loop Reflectors," Journal of Lightwave Technology 6(7), 1217-1224 (1988).

[2] Wang, Y., Zhao, C.-L., Dong, X., Kang, J., and Jin, S., “A fiber loop mirror temperature sensor demodulation technique using a long-period grating in a photonic crystal fiber and a band-pass filter," Review Of Scientific Instruments 82(7), art. no. 073101 (2011).

[3] Liu, Y., Liu, B., Feng, X., Zhang, W., Zhou, G., Yuan, S., Kai, G., and Dong, X., "High-birefringence fiber loop mirrors and their applications as sensors," Applied Optics 44(12), 2382-2390 (2005).

[4] Zhao, C-L., Yang, X., Lu, C., Jin, W., and Demokan, M.S., "Temperature-Insensitive Interferometer Using a Highly Birefringent Photonic Crystal Fiber Loop Mirror," IEEE Photonics Technology Letters 16(11), 2535-2537 (2004).

[5] Campbell, M., Zheng, G., Holmes-Smith, A.S., and Wallace, P.A., "A frequency-modulated continuous wave birefringent fiberoptic strain sensor based on a Sagnac ring configuration," Measurement Science and Technology 10(3), 218-224 (1999).

[6] Hatta, A.M., Semenova, Y., Wu, Q., and Farrell, G., "Strain sensor based on a pair of single-mode-multimode-single-mode fiber structures in a ratiometric power measurement scheme," Applied Optics 49(3), 536-541 (2010).

[7] Dong, B., Zhao, Q., Lvjun, F., Guo, T., Xue, L., Li, S., and Gu, H. "Liquid-level sensor with a high-birefringence-fiber loop mirror," Applied Optics 45(30), 7767-7771 (2006).

[8] Kim, K.-J., Yeom, S.-H., Kang, B.-H., Kim, D.-E., and Kang, S.-W., "Micro-Optic Mach-Zehnder Interferometric Sensor for UV Detection Using Photochromic Dye," Sensor Letters 9(1), 195-198, (2011).

[9] Silva, S., Frazão, O., Viegas, J., Ferreira, L.A., Araújo, F.M., Malcata, F.X., and Santos, J.L., "Temperature and strainindependent curvature sensor based on a singlemode/multimode fiber optic structure," Measurement Science and Technology 22(8), 085201 (2011).

[10] André, R.M., Marques, M.B., Roy, P., and Frazão, O., "Fiber Loop Mirror Using a Small Core Microstructured Fiber for Strain and Temperature Discrimination," IEEE Photonics Technology Letters 22(15), 1120-1122 (2010).

[11] R. M. Silva, A. Layeghi, M. I. Zibaii, H. Latifi J. L. Santos, O. Frazão, Theoretical and Experimental Results of High Birefringent Fiber Loop Mirror with an Output Port Probe, J. Lightw. Technol., 30 (8), (2012).

[12] M. Dahlem, J. L. Santos, L. A. Ferreira, F. M. Araújo, "Passive Interrogation of Low Finesse Fabry Pérot Cavities Using Fiber Bragg Gratings", IEEE Photon. Technol. Lett., (9), 990-992, (2006). 